Sammlung Metzler

Band 287 
Hartmut Eggert / Christine Garbe

\section{Literarische Sozialisation}

Verlag J.B. Metzler

Stuttgart - Weimar 
Die Deutsche Bibliothek - CIP-Einheitsaufnahme

\author{
Eggert, Hartmut: \\ Literarische Sozialisation / \\ Hartmut Eggert/Christine Garbe. - \\ Stuttgart; Weimar : Metzler 1995 \\ (Sammlung Metzler ; Bd. 287) \\ ISBN 978-3-476-10287-4 \\ NE: Garbe, Christine:; GT
}

ISBN 978-3-476-10287-4

ISBN 978-3-476-03984-2 (eBook)

DOI 10.1007/978-3-476-03984-2

ISSN 05583667

SM 287

Dieses Werk einschließlich aller seiner Teile ist urheberrechtlich geschützt. Jede Verwertung außerhalb der engen Grenzen des

Urheberrechtsgesetzes ist ohne Zustimmung des Verlages unzulässig und strafbar. Das gilt insbesondere für Vervielfältigungen, Übersetzungen, Mikroverfilmungen und die Einspeicherung und Verarbeitung in elektronischen Systemen.

(C) 1995 Springer-Verlag GmbH Deutschland

Ursprünglich erschienen bei J.B. Metzlersche Verlagsbuchhandlung und Carl Ernst Poeschel Verlag GmbH in Stuttgart 1995 


\section{Inhalt}

Vorwort

I. Grundlegungen 1

1. Literaturwissenschaft und literarische Sozialisation ....................................................... 1

2. Lesekonzepte und Lesefähigkeiten ………………...... 8

3. Sozialisationskonzepte und Lesekultur .................... 17

4. Entwicklung literarischen Verstehens ..................... 22

II. Historische Entwicklung der Theorieansätze....... 27

1. Theorie des Lesealters............................................... 27

2. Psychoanalytische Ansätze …………...................... 36

3. Soziologische Arbeiten ............................................ 43

III. Aktuelle Forschungsrichtungen ............................ 52

1. Kommunikationswissenschaftliche Forschung ........ 52

2. Lesebiographische Forschung / Fallstudien ...............59

3. Psychoanalytische Forschung ................................... 67

4. Kinder- und Jugendliteratur-Forschung ................. 71

IV. Geschlechtsspezifische Forschungsansätze ........... 76

1. Empirische Befunde zum Leseverhalten der Geschlechter .................................................... 77

2. Theoretische Erklärungsansätze ................................ 80

3. Die Diskussion um das Mädchenbuch .................... 85

V. Literarische Sozialisation in der Kindheit ............ 93

1. Familienkindheit - Lesekindheit im 18. und 19. Jahrhundert .................................. 93

2. Leselust und Lesemotivation in der Kindheit ......... 95

3. Von der Mündlichkeit zur Schriftlichkeit .............. 100

4. Das Märchen - eine kinderliterarische Gattung im Umbruch .................................................. 104

5. Familie und kindliches Lesen im Medienzeitalter 
VI. Literarische Sozialisation im Jugendalter

(Pubertät und Adoleszenz)

1. Jugend als geschichtliches Phänomen ................. 115

2. Von der ,Kulturpubertät ‘ zur `Medienjugend ‘...... 117

3. Pubertäre 'Lesesucht ‘...................................... 122

4. Lesen und adoleszente Identitätsbildung .............. 128

5. Jugendlektüre und Deutschunterricht ................ 135

6. Literarische Sozialisation im Medienverbund ....... 146

Zusammenfassende Ausblicke

Bibliographie

163

Personenregister

210

Angabe zu den Autoren

214 


\section{Vorwort}

Die Zukunft der literarischen Kultur wird davon mitgeprägt, welchen Gebrauch Kinder und Jugendliche des Medienzeitalters von ihr machen werden. Welche Beziehungen bestehen zwischen den kinderliterarischen Praktiken, jugendlichen Lektüregewohnheiten und der (späteren) literarischen Kultur der Erwachsenen?

Vor weniger als drei Jahrzehnten härte man die Beantwortung dieser Fragen ohne Zögern in die Zuständigkeit von , Literaturdidaktik verwiesen. Seit dem Aufstieg des Fernsehens zum Leitmedium in weiten Bevölkerungskreisen ist spätestens deutlich geworden, daß dieser fachlich begrenzende Blick nicht zureicht. Die Ausgriffe in andere Fachdisziplinen (wie Psychologie, Leseforschung, Kommunikationswissenschaft, Soziologie, Kinder- und Jugendliteraturforschung etc.) bedeuteten aber nicht nur einen Zugewinn an Wissen, sondern förderten die Entdeckung bis heute offener Frage- und Problemstellungen. Die inzwischen auch wissenschaftstheoretisch untermauerte Einsicht, daß mit mehr , Wissen ` proportional das,$N i c h t-W i s s e n$ ` wächst, zeitigte Forschungshaltungen, die eher Abgrenzungen als Integration betonen.

Unser Vorsatz, eine Einführung in ein Lehr- und Forschungsgebiet Literarische Sozialisation zu verfassen, die sich an einer neuen integrierenden Fragestellung orientiert, mußte davon geprägt sein, für unterschiedliche fachlich-methodische Ansätze offen zu sein, aber gleichzeitig einen focussierenden Blick zu entwickeln, der der Tendenz zur Autonomisierung von Einzelaspekten entgegenwirkt.

Manchmal sind wir bei diesem Unterfangen an Grenzen gestoßen, die Erwartungshaltungen an die Reihe Sammlung Metzler. Realien zur Literatur und daraus abgeleitete Vorgaben setzen. Erörternde Darstellungen, die im Horizont der Leserinnen und Leser Problembewußtsein stimulieren können, mußten häufiger zurücktreten gegenüber straffender Information und der Verweisung auf problemvertiefende Lektüre. Wir haben einen Aufbau in der Darstellung gewählt, der es nahelegt, einzelne Kapitel in ihrer Geschlossenheit zu lesen und das 
Buch in seinen gegliederten Einzelaspekten nicht als ,Nachschlagetext für Spezialforschung zu verwenden.

Schon bei der Durchsicht der Bibliographie wird man darauf stoßen, daß hier eine weitgehende Beschränkung auf Forschungen für den deutschsprachigen Raum vorliegt. Diese Eingrenzung hat eine Fundierung in der Sache, die uns beim gegenwärtigen Forschungsstand geboten schien: Wie aus unserer Darstellung deutlich werden kann, ist literarische Sozialisation stark geprägt von Wechselbeziehungen zwischen Bildungssystem, Eigenheiten der literarischen Tradition und kultureller Normen, Strukturen des literarischen Lebens und der allgemeinen Medienentwicklung. Die Probleme, die mit der Übertragung von Forschungsergebnissen aus anderen literarischkulturellen Kontexten gegeben sind, wollten wir bei dieser erstmals verfaßten Einführung möglichst gering halten, obwohl wir wissen, daß der skomparatistische Blick r die Konturen von literarischen Sozialisationen in der deutschen Tradition schärfer hervortreten lassen könnte. $\mathrm{Ob}$ die Unterschiede, die dem Kenner der literarischen Kulturen innerhalb Europas vertraut sind, sich unter den Bedingungen heutiger Medienstrukturen und der Internationalisierung gesellschaftlicher Entwicklung in der westlich-technologischen Zivilisation nivellieren, ist in der Forschung überhaupt erst angedacht, selbst wenn die Stimmen in der breiten publizistischen Öffentlichkeit schon entsprechende Gewißheiten verbreiten.

Die vorliegende Einführung wurde von uns gemeinsam konzipiert und redigiert, wir haben aber darauf verzichtet, Stilnuancen und unvermeidliche individuelle Wertungen, die aus der Erarbeitung einzelner Kapitel erwachsen sind, zu tilgen. Die Kapitel I-III wurden von Hartmut Eggert, die Kapitel IVVI von Christine Garbe verfaßt. An der technischen Herstellung des Typoskripts hat Marlene Stukowske mitgewirkt; Bettina Neuhaus war für die bibliographische Registratur, Ordnung und Überprüfung mitverantwortlich und Christiane Kemmler ihr bei ergänzender Recherche und Erarbeitung des Registers behilflich.

Berlin, November 1994

Hartmut Eggert/Christine Garbe 